\title{
ACOMPAÑAMIENTO MEDIACIONAL PARA DESARROLLAR LA COMPETENCIA ESCRITURAL DE ESTUDIANTES DE MAESTRÍA
}

\author{
Mediational Support to Develop \\ the Writing Competence of Master's Students
}

María Margarita Villegas ${ }^{1}$

margaritavillega3017@gmail.com

\section{Fredy González ${ }^{2}$}

fredygonzalez@hotmail.com

${ }^{1}$ Universidade Federal Rural do Semi-Árido. Brasil

${ }^{2}$ Universidade Federal de Rio Grande do Norte. Brasil

Recibido: 27/02/2021

Revisado: $17 / 03 / 2021$

Aprobado: 7/05/2021

Preprint: 1/06/2021

Publicado: 15/07/2021

\section{Resumen}

Se reportan procesos y estrategias utilizadas en el acompańamiento mediacional para desarrollar la competencia escritural de estudiantes de una maestría en enseñanza. Las teorías sobre la producción escrita y la escritura como actividad social (Bolívar, 2020; Carlino, 2005; Cassany \& Castellà, 2010; Freire, 1984; Gordillo-Alfonso, 2017), sobre la mediación y acompañamiento mediado (González, 2008; Villegas \& Hernández, 2017), y sobre la situación social de desarrollo y enfoque histórico cultural (Rodríguez-Arocho, 2020; Vygostky, 1966) sirvieron de soporte para realizar las interpretaciones correspondientes. Metodológicamente, se trata de un relato de experiencia producto del análisis y reflexión sobre los procesos y las estrategias de mediación que utilizó una profesora investigadora para formar en la escritura científica a 15 estudiantes de maestría de una universidad pública. Los resultados revelan que el acompañamiento mediacional, a través de varios procesos contextualizados (revisión documental y bibliográfica, escritura individual y reflexión colaborativa grupal, revisión mediada, indagación dialógica y participación sustentada), es fundamental para el desarrollo de la competencia escritural de investigadores en formación.

Palabras clave: escritura académica, estrategia de enseñanza, estrategia didáctica, maestría, mediación tutorial.

\section{Abstract}

Processes and strategies used in the mediational accompaniment to develop the writing competence of students at a master's degree in teaching are reported. Theories on written production and writing as a social activity (Bolívar, 2020; Carlino, 2005; Cassany \& Castellà, 2010; Freire, 1984; Gordillo-Alfonso, 2017), on mediation and mediated accompaniment (González, 2008; Villegas \& Hernández, 2017), and on the social situation of development and historical-cultural approach (Rodríguez-Arocho, 2020; Vygostky, 1966) served as support to make the corresponding interpretations. Methodologically, this is an experience account product of the analysis and reflection on the processes and mediation strategies used by a research professor to train 15 master's degree students at a public university in scientific writing. The results reveal that mediational support, through various contextualized processes (documentary and bibliographic review, individual writing and collaborative group reflection, mediated review, dialogic inquiry and sustained participation), is essential for the development of the writing competence of researchers in training.

Keywords: academic writing, didactic strategy, master's degree, mediated review, mediational support, teaching strategy, tutorial mediation. 


\section{Introducción}

El ser humano se constituye en relación con los otros y desde los otros, compartiendo prácticas y experiencias como sujeto sociocultural; en consecuencia, es producto de una historia y de contextos en los que es partícipe de prácticas socioculturales (Mendes \& Silva, 2017) que afectan su modo de ser y de actuar, así como sus maneras de pensar. Pero lo contrario también puede ser. El ser humano tiene la posibilidad de modificar su hábitat ya que, gracias a su sentido crítico, puede desarrollar un rol activo en la definición tanto de la dirección como de la forma en la que tendrán lugar sus acciones y las experiencias que lo irán moldeando a lo largo de toda su existencia vital; a esto algunos autores lo denominan "construcción social del sujeto" (Berger \& Luckmann, 1968; Braunstein, 1980; Sosa, 2010).

En relación con la noción de construcción social del sujeto singular, Ludwik Fleck, en su libro Génesis y desarrollo de un hecho cientifico (1986), afirma que la mente del sujeto individualmente considerado es producto de una mente colectiva generada en lo que este autor denomina "colectivo de pensamiento".

Próxima a esta idea de Fleck, en la perspectiva del enfoque histórico-cultural (EHC), se encuentra Rodríguez-Arocho (2020) cuando afirma que las estructuras cognitivas del individuo se conforman en el curso de su desarrollo ontogenético en el que su actividad práctica junto con la de otros sujetos y la comunicación entre ellos juegan un papel determinante (Rodríguez-Arocho, 2020, p. 126).

Por tanto, de acuerdo con el EHC, las actividades personales de los individuos están mediadas por la cultura en la que están inmersos. Además de Fleck (1986), esta relación entre cognición individual y cognición social ha sido formulada por Vygostky (1996), Roggoff (1993) y Wells (2004), entre otros autores.

La articulación individuo-sociedad es de singular importancia en el ámbito académico universitario, en el que se promueven la cooperación y la colaboración en la realización de las diferentes experiencias de aprendizaje, entre ellas las que implican la producción de trabajos escritos.

En efecto, como afirman Bellón y Cruz (2002), la escritura es una "construcción sociocultural compleja" que ha de ser comprendida como "un instrumento que media las relaciones de los sujetos culturales con sus contextos particulares y su vida interior. Ella les permite interactuar con otros sujetos culturales para crear otros universos" (Bellón \& Cruz, p. 58). Pero desde el punto de vista operativo, la escritura es una competencia individual y singular que se desarrolla con la mediación de otros, puesto que tal como lo señala Bolívar (2020), la escritura

[...] no se da en el vacío sino en contextos culturales en los que la investigación es el producto de interacción entre personas que junto con construir conocimiento se construyen a sí mismas discursivamente como grupo y como personas en los textos que escriben (Bolívar, 2020, p. 224).

Por otro lado, escribir y al mismo tiempo comprender lo que se escribe teniendo en cuenta tanto el para qué como el para quién se escribe —es decir, escribir y simultáneamente tener conciencia de lo que está siendo escrito- es una competencia escritural sofisticada, lo cual implica, de acuerdo con Lacon y Ortega (2008), que el escritor "construya una representación adecuada de la tarea de escritura en la memoria, lo que supone el conocimiento de estrategias de producción y su uso consciente" (Lacon \& Ortega, 2008, p. 232).

Es precisamente esta capacidad escritural la que deben desarrollar los estudiantes universitarios en general, y en particular quienes realizan estudios de posgrado. De allí la importancia de la mediación en el desarrollo de las competencias escriturales de los estudiantes de posgrado. Tal mediación se entiende como estrategias

[...] que resaltan la actividad del sujeto en su propio aprendizaje y los procesos caracterizados por la atribución de significado y sentido a la experiencia vivida, la interacción del sujeto con otros actores sociales en el proceso educativo, el diálogo, la comunicación y la colaboración (Rodríguez-Arocho, 2020, p. 2). 
La mediación es necesaria si se desea que los estudiantes de posgrado incrementen su competencia para producir textos escritos, lo que, en general, parece no estar ocurriendo. En efecto, los resultados de investigaciones como la de Triana-Murillo (2015) denotan que el 85\% de los estudiantes de posgrado de una institución de Educación Superior colombiana se ubican en niveles deficiente a regular.

Una situación análoga también se da en México; así lo evidencian Rodríguez y Leal (2017) al reflexionar sobre "las complicaciones con la escritura académica que enfrentan estudiantes durante la construcción de sus documentos para obtener el grado académico":

[...] la falta de conocimientos sobre el proceso de producción textual que exige esta práctica escritural y la escasa experiencia de los alumnos en la elaboración de este tipo de escritos dificultan la construcción de los documentos y obstaculizan su conclusión (Rodríguez \& Leal, 2017, p. 224).

Sin embargo, la situación antes descrita puede ser modificada si se desarrollan estrategias adecuadas. Así parecen confirmarlo estudios como el de Bosio (2018), quien afirma que

[...] la modelización y la explicitación del proceso de escritura, el entrenamiento estratégico sistemático en géneros académicos y un conjunto de criterios de evaluación coherentes con dicha explicitación contribuyen al incremento de la calidad de la producción escrita en el nivel de posgrado (Bosio, 2018, p. 737).

Parece ser, entonces, que la escasa mediación social es uno de los factores que afecta la producción escrita de los estudiantes de posgrado, pues regularmente trabajan en solitario, sin la preparación adecuada $y$ sin un contexto favorecedor (Carlino, 2005). Las dificultades para escribir que manifiestan estos estudiantes tienen incidencia directa en las posibilidades de culminar con éxito sus estudios. Con frecuencia, incluso habiendo completado toda su carga académica en cuanto a disciplinas o asignaturas que deben cursar, no logran producir en el tiempo previsto el trabajo de conclusión, llámese trabajo de grado de maestría o tesis de doctorado. Este fenómeno ha sido denominado "todo menos tesis" (TMT) y ha motivado interesantes estudios como los de Valarino (1991), Abreu (2015) y Gascón (2008), entre otros.

En su reciente estudio sobre los factores asociados a la elaboración de trabajos de maestría y tesis de doctorado, realizado en una universidad suramericana, Soto-Figueredo (2020) analizó un periodo de 10 años (2004-2014) y encontró que "El principal factor $(22 \%)$ que impide la realización de la tesis es la cuestión metodológica (el o la tesista no sabe cómo hacer la tesis, tiene problemas con la escritura) (Soto-Figueredo, 2020, p. 1) (cursivas añadidas).

Muchas personas que emprenden estudios de posgrado no han desarrollado previamente habilidades para producir trabajos académicos escritos, puesto que la escritura no ha sido asumida como una actividad social sino como una relación solitaria con el texto (Carlino, 2005) y con el docente evaluador, sin otra intención que aprobar la disciplina que esté siendo cursada.

Así, en cuanto a las dificultades de la escritura académica, Hernández-Zamora (2009) identifica las siguientes: (a) desconocimiento de la amplia variedad de textos académicos (expositivos, argumentativos, etc.); (b) carencia de estrategias para escribir (planificación, revisión a posteriori); (c) carencias en cuanto a redacción, ortografía, puntuación; (d) limitaciones en comprensión de lo leído; y (e) dificultades para plantear y argumentar posiciones personales.

Las anteriores dificultades también fueron constatadas en Brasil por la autora del presente artículo, quien, actuando como profesora en una maestría interinstitucional en Enseñanza, verificó que sus estudiantes tenían limitaciones para realizar las tareas que implicaban la elaboración de producciones escritas.

Fue esta constatación la que motivó la realización del presente artículo cuyo objetivo principal fue coadyuvar al desarrollo de la competencia escritural de 15 estudiantes cursantes de la maestría en Enseńanza, acompañándolos mediacionalmente en la producción de sus textos escritos. 


\section{Revisión de la literatura}

La premisa teórica fundamental asumida en este estudio es que la escritura constituye "una práctica discursiva socialmente situada" (Hernández-Zamora, 2009); en este sentido se suscribe el planteamiento de este autor que afirma que

Los textos escritos no son, desde este punto de vista, artefactos lingüísticos autónomos, separados de los contextos sociales de interacción, sino piezas de lenguaje contextualmente situadas. Este punto de vista ha sido reafirmado por los estudios socioculturales de la alfabetización, que han caracterizado la escritura como una práctica socialmente situada [...] la escritura académica [puede ser entendida] como un proceso dialógico que implica establecer identidades sociales mediante la apropiación de las palabras de otros [...] (Hernández-Zamora, 2009; 19-20) (cursivas en el original).

Con base en lo anterior, el repertorio de coordenadas teórico-conceptuales de referencia (RCT-CR) (González, 2017) está compuesto por las nociones descritas a continuación.

\subsection{La escritura como construcción social}

De acuerdo con Cassany \& Castellà (2010), para la producción de textos escritos ya no es suficiente estar alfabetizado en el sentido tradicional del término, sino que es algo más trascendente, tal como lo expone Freire (1984), para quien la alfabetización es el proceso de comprender el mundo, ir más allá de la decodificación de textos, y así entender el lenguaje y la realidad como fenómenos sociales.

Desde esa perspectiva, el proceso de producción escrita se amplía hacia las nociones de cultura letrada y letramento; es decir, aquellas implicadas en el desarrollo de buenas prácticas lecto-escriturales. La literacidad incluye

[...] el dominio y el uso del código alfabético, la construcción receptiva y productiva de textos, el conocimiento y el uso de las funciones y los propósitos de los diferentes géneros discursivos de cada ámbito social, los roles que adoptan el lector y el autor, los valores sociales asociados con estos roles (identidad, estatus, posición social), el conocimiento que se construye en estos textos y que circula en la comunidad, la representación del mundo que transmiten, etc. (Cassany \& Castellà, 2010, p. 354).

De ese modo, la construcción de la lengua escrita y oral se concibe como un fenómeno más global ligado a una cultura, a valores sociales y a mecanismos de constitución de educación y formación.

En esa perspectiva social de la producción escrita, según lo afirma Bolívar (2020), es importante conocer las tradiciones discursivas - junto con sus patrones de comunicación y de elaboración de textos- de la comunidad para la cual se escribe, ya que conocer, leer y escribir son procesos que se desarrollan en contextos de prácticas mediadas culturalmente (Vygotsky, 1996); por eso, Freire (1984) siempre alegaba que el conocimiento, la lectura y la escritura son procesos que se dan en conexión con el mundo asumiendo que las experiencias vividas ocurren en escenarios donde se "es" y ocurre "siendo", y no escapan de un lugar y un tiempo en un contexto de ser-en-el-mundo.

Por ende, siguiendo las ideas de Heidegger (1976, 1986), es necesario reflexionar sobre ese ser-enel-mundo como una relación permanentemente reforzada por nuestro ser con otros, con quienes compartimos el mismo mundo y en el cual se producen interacciones recíprocas que contribuyen a la modificación mutua de modos de pensar y de actuar, lo que cual es fundamental en los procesos formativos propios de las acciones educativas.

\subsection{Papel de las prácticas socioculturales en la producción de conocimientos}

De acuerdo con Caballero-Merlo (2016), la producción de conocimientos en el campo de las ciencias sociales acontece bajo determinadas condiciones contextuales; para ello, este autor hace referencia a los tres contextos de emergencia del conocimiento científico propuestos por Reichenbach (1961, pp. 6-7): el de descubrimiento, el de justificación y el de aplicación. 
En cuanto al significado del vocablo contexto, Rogoff (1993) lo entiende como una red de relaciones entrelazadas que estructuran los significados y los sentidos que las personas asignan a sus acciones. De acuerdo con esta autora, no hay situaciones libres de contexto ni habilidades descontextualizadas. Por ende, se aprende en situaciones sociales situadas.

Este carácter socialmente situado de las acciones humanas individuales o colectivas es destacado por Vygotsky (1996) al indicar la naturaleza sociogenética de la actividad cognitiva, aludiendo a la esfera motivacional de la conciencia, mediada por las inclinaciones, necesidades e intereses individuales (Vygotski, 1993, p. 342). Así, trascendiendo el concepto de zona de desarrollo próximo (aquello que el individuo puede ejecutar si cuenta con el apoyo de otro), es el propio Vygotsky quien introduce el concepto de situaciones sociales de desarrollo (Vygotsky, en Rodríguez-Arocho, 2020), en las cuales tanto las vivencias como las experiencias subjetivas de los individuos son pensadas e interpretadas en función de las particularidades de un ambiente social y cambian y se reconfiguran en el curso del desarrollo humano.

Con base en lo expuesto, puede ser inferido que el conocimiento que una persona construye es tanto causa como efecto de su inserción en el mundo heideggeriano y también y principalmente en el contexto sociocultural vygotskiano.

\subsection{La escritura científica como una práctica sociocultural singular que requiere apoyo media- cional institucional}

Como fue afirmado por Drucker (1993), estamos inmersos en una sociedad del conocimiento en la cual tiene cada vez más valor el modo de presentación y el intercambio de saberes. Particularmente, en el contexto universitario, en relación con la escritura es muy relevante la producción de textos científicos (Gordillo-Alfonso, 2017), y es el principal requisito para evaluar el desempeño académico de los estudiantes, especialmente de quienes realizan estudios de posgrado.
Gordillo-Alfonso (2017) admite que ser docente e investigador y escribir un artículo científico es una tarea que demanda tiempo, esfuerzo, formación y dedicación por parte de quien la asume, y constituye uno de los desafíos más exigentes que deben ser superados por los estudiantes que realizan estudios de posgrado pues generalmente, como lo indica Carlino (2005), es un proceso que se lleva a cabo en solitario, “[...] centrado en temas desvinculados de los temas de sus compañeros y carente de apoyo institucional y [como toda actividad social] [...] requiere no solo un compromiso personal del aprendiz sino un soporte [mediacional] externo [...]" (Carlino, 2005, p. 418).

En ese contexto, uno de los aspectos que las instituciones deben reconocer es que la escritura es una tarea intelectualmente exigente (TIE) (González, 1998), cuya ejecución requiere prácticas que permitan la realización de actividades que exijan la producción de textos académicos escritos, con lo que se constituye en un reto tanto para los estudiantes como para sus docentes, ya que "la producción de textos académicos implica competencias discursivas y comunicativas y procesos cognitivos específicos ante los cuales los estudiantes suelen presentar problemas" (Roa-Rodríguez, 2014, p. 70), especialmente en los ámbitos académicos cuando los docentes o los estudiantes deben realizar actividades que pueden ser caracterizadas como TIE.

La escritura de textos científicos puede ser concebida como TIE, ya que es una acción compleja que no se limita a considerar solamente aspectos y procesos cognitivos, sino que además abarca prácticas sociales enraizadas en la cultura de la cual forma parte el escritor (Bolívar, 2020; Carlino, 2005; Cassany \& Castellà, 2010); ello explica de algún modo las dificultades que deben superar quienes desean publicar los resultados de alguna investigación, como es el caso de los estudiantes de posgrado, a quienes se les exige escribir textos académicos susceptibles de ser publicados como artículos en revistas científicas.

Existen evidencias que confirman que las limitaciones para escribir constituyen un obstáculo para el aprendizaje; así lo reconoce Rodríguez (2013), quien 
afirma que tales dificultades pueden llegar a causar en los estudiantes "[...] bloqueos serios cuando tienen que utilizar la escritura como medio de expresión de lo que previamente han memorizado, o lo que han tenido que sintetizar, crear, inventar o resumir (Rodríguez, 2013, p. 7).

El caso de los estudiantes que emprenden estudios de posgrado debido, entre otras razones, al cambio de su rol, tal como lo señalan Nothstein \& Valente (2016), a continuación:

[...] al ingresar en el IV nivel de su educación (maestría), el alumno pasa de haber ejercido el rol de lector especializado, de receptor activo y crítico de los saberes que fue integrando y sistematizando a lo largo de su formación, al de ser productor de conocimientos nuevos e iniciarse en el proceso de construcción de su espacio propio en el campo intelectual o disciplinar (Nothstein \& Valente, 2016, p. 141).

Nothstein y Valente (2016) agregan que comenzar a estudiar un posgrado implica ingresar en una nueva comunidad de pensamiento, en la que los estudiantes se ven conminados a generar "[...] producciones escritas y orales con las que no necesariamente han estado familiarizados durante su formación previa” (p. 129); tal ingreso demanda un proceso de inmersión en una cultura formativa distinta de aquella de la cual provienen los estudiantes (la propia de los estudios preuniversitarios y universitarios), en la que la escritura no es una actividad cotidiana. Por ello, al verse conminados a escribir en posgrado, los estudiantes tienen una serie de carencias escriturales. Entre dichas carencias, Hernández-Zamora (2009) ha inventariado las siguientes:

1. Falta o uso incorrecto de citas literales o referencias bibliográficas.

2. Aparente ausencia de planeación y revisión antes, durante y después de escribir el texto.

3. Mezcla de fragmentos expositivos, analíticos e interpretativos (es difícil distinguir las ideas de los autores referidos y los comentarios propios de los estudiantes).

4. Dificultad para: a. distinguir qué preguntas se hacen al texto que se está exponiendo o refiriendo, qué preguntas surgen del texto y qué juicios se emiten sobre el texto.

b. estructurar un discurso propio y para insertar en este contexto citas textuales y datos empíricos.

c. leer, entender y reconstruir los textos teóricos.

d. recuperar información teórica y empírica, ya sea para sustentar una postura personal o para exponer una postura contraria.

e. sintetizar (es difícil escribir poco, porque para hacerlo uno necesita entender lo que se lee).

5. Mezcla de lenguaje coloquial con léxico especializado.

6. Ante la insistencia de que se planteen sus propias preguntas, proliferación de listas interminables de preguntas sin ninguna jerarquización ni respuestas probables.

7. Errores ortográficos [sic, faltas de ortografía], sintaxis o redacción confusa, discordancia gramatical (sustantivo/verbo/artículo).

8. Naturaleza del texto solicitado: muchos estudiantes escriben descripciones monográficas, cuando lo que se les solicitó fue escribir sus opiniones (texto argumentativo); otros son prolíficos en narrativas personales, cuando lo que se pidió fue una respuesta a un libro o artículo específico. En resumen, la mayoría de los estudiantes escriben normalmente "textos híbridos", cuyo género es difícil de determinar, y cuya lectura se vuelve muy difícil (lenta, tortuosa) (Hernández-Zamora, 2009, pp. 23-24).

Otros autores también han identificado obstáculos para el desarrollo de la escritura científica, entre ellos Bolívar (2020), quien señala la existencia de varios tipos de texto acerca de los cuales los estudiantes no tienen (in)formación alguna; otro obstáculo es la limitada información que algunas revistas científicas ofrecen sobre los criterios específicos que deben satisfacer quienes aspiren a ser autores.

Para superar estos y otros obstáculos, Bolívar (2020) sugiere la creación de escenarios más formales para la producción de textos científicos puesto que, como 
expone Maingueneau (2002), la producción escrita "supone procesos cognitivos específicos y un posicionamiento enunciativo particular a partir del cual el estudiante debe construir un nuevo ethos en tanto que productor de nuevos saberes" (Maingueneau, 2002; citado en Nothstein \& Valente, 2016, p. 129). Lo anterior significa que para escribir textos y artículos científicos se requiere una formación idónea, la cual no se desarrolla de modo espontáneo, sino que es propiciada mediante el ingreso en una comunidad de investigadores desde etapas tempranas de la escolaridad.

\subsection{La mediación social como estrategia para el desenvolvimiento del pensamiento individual}

Una opción para superar los obstáculos que los estudiantes de posgrado enfrentan para realizar sus producciones escritas es desarrollar un sistema de mediación tutorial (SMT) (González, 1996) para acompañarlos durante las diferentes etapas del proceso de una producción escrita. Para el caso de los estudiantes de posgrado, este sistema busca apoyarlos con instancias de mediación presididas por el respeto y la horizontalidad entre personas que son profesionales (Villegas \& Hernández, 2017).

Además, el SMT debe propiciar la generación de instancias de indagación dialógica (Villegas \& Hernández, 2017) en las que los propios estudiantes, todos juntos, reflexionen sobre los siguientes aspectos: dificultades que pudiesen estar confrontando; aspectos que requieren ser mejorados, y conocimientos que deben ser construidos. Con todo ello se aspira contribuir al incremento de su conciencia acerca de las demandas propias de la producción escrita de textos académicos, tales como artículos, trabajos de grado de maestría o tesis de doctorado.

Otra característica que ha de tener el SMT es la posibilidad de que produzcan colaborativamente textos escritos, lo cual se ha evidenciado como una estrategia idónea para el desarrollo de competencias escriturales, como lo muestran Castelló, González e Iñesta (2010), quienes encontraron que esta acción propició mejoras en la producción de textos científicos de los participantes de un programa de doctorado español en Investigación Psicológica, lo cual se podría transferir a otros programas de posgrado.

\section{Metodología}

Diseño. El trabajo consiste en un relato de experiencia de mediación de la competencia para la escritura científica desarrollada durante el proceso de enseñanza aprendizaje de una asignatura administrada para 15 profesores pertenecientes a una maestría de enseñanza que se desarrolla en una universidad pública brasilera. La autora principal del presente artículo actuó como facilitadora y, al mismo tiempo, como investigadora.

Escenario generatriz de interacciones estudiantes-participantes y profesora-Investigadora. Las actividades de mediación se llevaron a cabo en 14 encuentros semanales, con una duración promedio de 270 minutos (encuentros de clase entre los estudiantes y la profesora/investigadora), en formato sincrónico y remoto, mediados tecnológicamente usando la plataforma GoogleMeet debido a las medidas de distanciamiento físico oficialmente ordenadas como disposición de bioseguridad a fin de evitar aglomeraciones y reducir la propagación del virus causante del COVID-19. Además de estos encuentros, se usaron otras vías de comunicación (llamadas telefónicas, mensajes a través de WhatsApp y de correo electrónico) que permitieron una comunicación fluida, constante y oportuna de los estudiantes con la profesora para el esclarecimiento de dudas, el ofrecimiento de orientaciones y el aporte de retroalimentación.

Prácticas de escritura. Para estimular su producción escrita, a los estudiantes se les instruyó para que escribieran informes sobre la lectura de artículos y otros documentos contentivos de los fundamentos teóricos de la asignatura.

Participaciones sustentadas. Consistían en intervenciones orales de los estudiantes llevadas a cabo durante los encuentros síncronos, las cuales, con el fin de evitar la exposición de ideas que no tuviesen relación con el contenido de la asignatura pautado para ser discutido en el encuentro, debían estar basadas 


\section{VILLEGAS • GONZÁLEZ}

Acompañamiento mediacional para desarrollar la competencia escritural de estudiantes...

en los informes de lectura previamente escritos y las intervenciones debían estar apoyadas en los aspectos planteados por los autores estudiados.

Escritura de una narrativa autobiográfica. A partir del séptimo encuentro, a los estudiantes se les instruyó para que escribieran un texto en el cual narraran las situaciones personales, emocionales, físicas, académicas y profesionales que hubiesen vivido como consecuencia de las medidas tomadas por el Gobierno brasileño para tratar de controlar la propagación del COVID-19. La escritura de este texto se constituyó en el trabajo evaluativo final de la asignatura cuya fecha de entrega fue prevista para el encuentro 14 . Fueron escritos 15 textos, uno por cada estudiante.

Los textos debían tener carácter individual y hacer referencia a circunstancias de su vida cotidiana en el contexto de la pandemia. Por tanto, era una escritura en primera persona sobre los fenómenos que más le hubieran impactado en sus roles de profesor de escuela y estudiante de posgrado.

Los textos se fueron escribiendo progresivamente; desde el octavo hasta el decimotercer encuentro se socializaron y discutieron colectivamente, prestando atención a los siguientes aspectos: características generales del texto, dificultades experimentadas por cada estudiante para escribirlo, y orientaciones para mejorarlos. Además, los textos fueron revisados y retro informados por escrito por la profesora varias veces hasta que alcanzaron el nivel suficiente como para ser publicados como capítulos de libro. Algunos textos se revisaron hasta ocho veces.

Por tratarse de una narrativa sobre la vida cotidiana de los estudiantes en el contexto de la pandemia del COVID-19, se acordó que el texto tendría la siguiente estructura: (a) introducción con la exposición del contexto del asunto motivador y las preguntas, objetivos o propósitos de la exposición a realizar; (b) exposición de la experiencia personal vivida, contextualizándola e interpretándola a partir de algunas de las teorías desarrolladas en la asignatura; (c) conclusiones: síntesis de las reflexiones derivadas a partir del asunto motivador y coherente con la totalidad del cuerpo del texto producido; y (d) cierre: reflexiones sobre el significado de la experiencia personal vivenciada durante la producción del texto escrito solicitado.

Los criterios de calidad considerados en la evaluación del texto fueron los siguientes:

1. Respeto a las normas convencionalmente aceptadas para la escritura de textos académicos, principalmente las relativas al uso correcto de citas literales y referencias bibliográficas.

2. Descripción del fenómeno que es objeto de interés, con apoyo en evidencias empíricas, emisión de juicios de forma idónea, realización de análisis e interpretaciones basadas en fundamentos teóricos pertinentes a su naturaleza y área.

3. Definición y profundización de la caracterización y el análisis de ciertos fenómenos (en este caso, de la vida cotidiana de los autores) realizando interpretaciones con apoyo en conceptos pertinentes y articulados con la naturaleza de la realidad especifica estudiada.

4. Originalidad de los planteamientos realizados a través de un discurso propio y apoyado en las referencias teóricas necesarias y con datos empíricos idóneos.

5. Estructuración que permita al lector sumergirse en el texto narrado y percibir la totalidad del asunto desarrollado, manteniendo la coherencia desde el inicio hasta el final del texto.

Mediación de la escritura de los participantes. El proceso de elaboración de los textos individuales de los participantes fue acompańado por la profesora-investigadora aplicando la estrategia denominada acompañamiento mediacional formativo de la competencia escritural, cuya dinámica se describe a continuación.

Estrategia de acompañamiento mediacional puesta en juego.

Lectura crítica de textos previamente asignados (generalmente artículos publicados en revistas acreditadas), elaboración de un breve informe de lectura 
(dos páginas como máximo) escogiendo libremente las ideas contenidas en el texto leído que fueran de su interés para ser debatidas en clase.

Socialización (lectura) de los Informes de lectura. Durante la clase, al llegar su turno cada estudiante expone sus ideas sobre el texto leído, las cuales son enriquecidas en el debate con los restantes miembros del grupo quienes comparten sus interpretaciones, formulan preguntas y hacen sugestiones.

Luego de finalizada la clase, cada estudiante envía (vía correo-e) su respectivo informe de lectura a la profesora, quien lo revisa y lo retorna al estudiante con su correspondiente revisión.

Dicha revisión está compuesta por dos partes, una caracterizada por comentarios al margen sobre observaciones de asuntos referidos a normas de la escritura y sobre vacíos observados en la estructuración de la redacción; otra, con un párrafo en la parte final del texto en el cual la profesora comunica su apreciación global sobre el texto, indicando aspectos favorables observados, necesidades de mejora, tanto en relación con errores conceptuales como en la organización y adecuación a las competencias de la escritura científica, cerrando con frases finales orientadas a estimular el esfuerzo sostenido para mejorar las próximas producciones, como una de las medidas más propicias.

\section{Resultados y discusión}

Como primer resultado de la aplicación de la estrategia de formación para la escritura académica es necesario indicar que todos los estudiantes participantes de este estudio, unos más que otros, tuvieron las dificultades indicadas por los autores antes referidos (Bolívar, 2020; Carlino, 2005; Hernández-Zamora, 2009; Nothstein \& Valente, 2016), entre las cuales se destacan las siguientes: poca familiaridad con el tipo de texto, desconocimiento de las características del tipo de texto, limitaciones para exponer sus propias ideas y argumentar frente a las ideas de los autores leídos, dificultades para redactar con un discurso propio, de forma coherente y con apego a las normas de la escritura académica.
Así mismo, se observó que los estudiantes poseían frente a la escritura la creencia según la cual esta es una habilidad innata, y por ello no la concebían como resultado de un esfuerzo constante y persistente; también concebían el proceso de escribir y los escritos como una actividad racional, libre de la subjetividad del lector y autor, cuyo producto no podía ser cuestionado luego. En este sentido, usaron las siguientes expresiones: "Yo no puedo cuestionar lo que está escrito. Si ese autor lo dice es porque es verdad", "Para escribir yo creo que se nace con la habilidad".

El acompañamiento mediacional se desarrolló con la intención de apoyar a los estudiantes de posgrado en el mejoramiento de sus habilidades para escribir textos académicos. Compartir las estrategias usadas para atender las necesidades presentadas por ellos es la finalidad principal del presente trabajo, el cual se sustenta en la perspectiva mediacional suscrita por Rodríguez (2020), quien afirma que:

La mediación pedagógica es una estrategia para la educación cognitiva cuya práctica se origina y desarrolla en enfoques que resaltan la actividad del sujeto en su propio aprendizaje y los procesos caracterizados por la atribución de significado y sentido a la experiencia vivida, la interacción del sujeto con otros actores sociales en el proceso educativo, el diálogo, la comunicación y la colaboración (Rodríguez, 2020, p. 2).

Los procesos constitutivos de la estrategia de mediación puesta en juego en el presente trabajo y a la que hemos denominado acompañamiento mediacional formativo de la competencia escritural se describen a continuación.

1. Revisión documental y bibliográfica, la cual vino a ser la actividad inicial impulsora para analizar y reflexionar sobre conceptos, principios, modos de comunicación escrita sobre las teorías de investigación cualitativa vinculados con los términos como sujeto, objeto, realidad, experiencias, vivencias, fenomenología y contextos, entre otros, con la participación de los estudiantes. 


\section{VILLEGAS • GONZÁLEZ}

Acompañamiento mediacional para desarrollar la competencia escritural de estudiantes...

2. Escritura de informes basados en la lectura de documentos (artículos o capitulos de libros) desarrollados de acuerdo con el interés particular del estudiante (v.g., lo que más le llamó la atención del documento leído) y respetando las normas de escritura convencionalmente aceptadas en Brasil.

3. Socialización oral: durante los encuentros sincrónicos, cada estudiante realizaba una exposición oral acerca del contenido del documento leído y del informe de lectura escrito.

4. Reflexión colaborativa grupal. Esta también se realizaba durante los encuentros sincrónicos; eran sesiones de discusión sobre diversas perspectivas de interpretación a fin de elaborar una síntesis de los conceptos y procesos tratados durante el encuentro. De ese modo, todos se daban cuenta de cuáles de sus ideas previas o nociones sobre determinado tema podían cambiar y cómo complementar sus conocimientos iniciales sobre el tema.

Los procesos anteriores se articulan en una estrategia conformada por las siguientes técnicas:

1. Revisión mediada. Se hizo en tres modalidades.

a. Autorrevisión. El estudiante relee su propio texto y, luego de participar en las discusiones propiciadas en el encuentro sincrónico, el estudiante se compromete a terminar de escribir su texto incorporando lo aprendido en la discusión.

b. Revisión colaborativa del texto entre los propios estudiantes para apoyarse mutuamente en la mejora de la producción.

c. Revisión de la profesora con retroinformación destacando tanto los aspectos positivos como aquellos que debían ser mejorados en relación con el contenido, la estructura del texto y las normas de la escritura académica.

2. Indagación dialógica. Esta actividad se dio en aquellos momentos en los que los estudiantes decían no comprender las orientaciones específicas para la adecuada escritura, lo cual se evidenciaba en los errores y fallas recurrentes en la escritura. Algunas de las fallas más frecuentes estaban asociadas con la emisión de juicios sin fundamentos en las investigaciones previas y carentes de evidencia científica. Para intentar subsanar estas fallas se consideró adecuado revisarlas con los propios estudiantes, haciéndoles preguntas y dialogando con ellos sobre las características que deben satisfacer los textos académicos, sobre cómo deben ser reportados los resultados vinculándolos con las fuentes de referencia, sobre la construcción de argumentos apoyados en el análisis de la información recaudada, y sobre cómo debe ser escrito el informe de lo que se haya leído.

3. Participación sustentada y activa de los estudiantes en cada clase con base en lecturas realizadas previamente sobre textos ya escogidos para que la participación en clase esté centrada en un mismo tema. En este momento de la clase los estudiantes son los protagonistas, pues el papel de la profesora se limita a moderar-mediar la participación a través de preguntas y de solicitudes de participaciones de otros estudiantes, que pueden complementar el tema en discusión con sus opiniones. Así, los estudiantes llegan por sí mismos a una interpretación más idónea de lo que estaban discutiendo, y pueden apropiarse una mejor elaboración lingüística y conceptual del asunto discutido.

\section{Reflexiones finales}

La experiencia desarrollada con este grupo de estudiantes de maestría en enseñanza permite afirmar que los procesos de mediación de revisión bibliográfica y documental, escritura individual y socialización oral de los avances y limitaciones, junto con las estrategias de revisión mediada, indagación dialógica y participación sustentaba, propiciaron un contexto idóneo para contribuir con el desarrollo de su habilidad para escribir textos académicos como los que son exigidos en los estudios de posgrado. Todos los estudiantes que participaron en esta experiencia lograron 
producir sus respectivos textos, los cuales, al momento de la escritura de este artículo, ya se encuentran en proceso de edición para formar parte de un libro que será publicado a mediados de este año 2021.

También se constata que uno de los resultados más importante fue la modificación de la actitud frente a la escritura de investigación, al abandonar la concepción inicial de percibirla como una actividad desprendida de la subjetividad del autor y con una visión claramente racional positivista para pasar a concebirla como una actividad social e interrelacional centrada en el esfuerzo colaborativo y mediado con otros y en la relación con los otros.

Además, la revisión bibliográfica nos permitió comprender que no es suficiente formar a través de la administración de asignaturas o cursos, sino que se requiere la constitución de un cuerpo pedagógico de docentes investigadores en el cual los estudiantes se inserten para apoyar las experiencias de investigación desarrolladas por las estudiantes en prácticas de mediación social.

\section{Referencias bibliográficas}

Abreu, J. L. (2015). Síndrome Todo Menos Tesis (TMT). Daena: International Journal of Good Conscience, 10(2), 246-259.

https://r.issu.edu.do/l?l=1067233N

Bellón-Morales, C., \& Cruz-Arias, M. del P. (2002). La escritura como actividad sociocultural compleja en el aula de transición: avances teóricos. Enunciación, 7(1), 57-63. https://r.issu.edu.do/l?1=10832xMi

Berger, P., \& Luckmann, T. (1968). La construcción social de la realidad. Buenos aires: Amorrortu.

Bolívar, A. (2020). La escritura de un buen artículo científico en educación y el entrenamiento de profesores universitarios en el discurso académico. Paradigma, 222-250.

https://doi.org/10.37618/PARADIGMA.1011-2251.2020.p222-250.id875

Bosio, I. V. (2018). ¿Podemos mejorar la calidad de la escritura en el posgrado? Algunas respuestas a partir de un proceso de investigación-acción. Revista
Brasileira de Linguistica Aplicada, 18(4), 737-769. https://doi.org/10.1590/1984-6398201812959

Braunstein, N. (1980). Psiquiatría, teoría del sujeto, psicoanálisis (hacia Lacan). México D.F.: Siglo Veintiuno.

Caballero-Merlo, J. N. (2016). Condiciones sociales de producción de conocimiento, el nosotros excluyente (ore), y las posibilidades del desarrollo de la comunidad científica en ciencias sociales en Paraguay. Revista Cientifica de la UCSA, 3(1), 47-66.

https://r.issu.edu.do/l?l=10674tVA

Carlino, P. (2005). ¿Por qué no se completan las tesis en los postgrados? Obstáculos percibidos por maestrandos en curso y magistri exitosos. Educere, Revista Venezolana de Educación, 9(30), 415-420.

https://r.issu.edu.do/l?l=10675oPZ

Caro, S. (2015). La lectura académica. Estrategias didácticas para el abordaje de la lectura en la universidad. Reflexión Académica en Diseño \& Comunicación. XXIII Jornadas de Reflexión Académica en Diseño y Comunicación (pp. 96-99). Universidad de Palermo. https://r.issu.edu.do/l?l=10676HMZ

Cassany, D., \& Castellà, J. (2010). Aproximación a la literacidad crítica. Perspectiva, Florianópolis, 28(2), 353-374.

https://doi.org/10.5007/2175-795X.2010v28n2p353

Castelló, M., González, D., \& Iñesta, A. (2010). La regulación de la escritura académica en el doctorado: el impacto de la revisión colaborativa en los textos. Revista Española de Pedagogía, 68(247), 521-537. https://r.issu.edu.do/l?l=10677CM6

Drucker, P. F. (1993). La sociedad poscapitalista. Traducción de María Isabel Merino Sánchez. Buenos Aires: Sudamericana.

Fleck, L. (1986). Génesis y desarrollo de un hecho cientifico. Madrid: Alianza.

Freire, P. (1984). La importancia de leer y el proceso de liberación. México: Siglo Veintiuno.

Gascón, Y. (2008). El síndrome de todo menos tesis "TMT" como factor influyente en la labor 
investigativa. Revista Copérnico, 5(9), 46-57. https://r.issu.edu.do/l?1=106787wW

González, F. (1998). Metacognición y tareas intelectualmente exigentes: el caso de la resolución de problemas matemáticos. Zetetiké, 6(9), 59-88. https:// doi.org/10.20396/zet.v6i9.8646808

González, F. (2008). Sistema de mediación tutorial. En F. González (Ed.), Modelos didácticos de base cognitiva (pp. 13-36). Venezuela: Ediciones del Centro de Investigaciones Educacionales Paradigma (CIEP).

González, F. (2014). Dificultades en la realización de trabajos de investigación: cómo afrontarlas. Práxis Educacional, 11(18), 275-300.

https://r.issu.edu.do/l?1=106795Sx

González, F. (2017). Repertorio de coordenadas teórico-conceptuales de referencia (RCT-CR) en las tesis del primer doctorado en Educación Matemática de Venezuela. En FESPM, Federación Española de Sociedades de Profesores de Matemáticas (Ed.), VIII Congreso Iberoamericano de Educación Matemática (pp. 69-77). Madrid: FESPM.

https://r.issu.edu.do/l?l=10680fD3

Gordillo-Alfonso, A. (2017). La escritura cientifica: una revisión temática. Signo y Pensamiento, $X X X$ $V I(71)$, 52-64. https://r.issu.edu.do/l?l=10681rKM

Heidegger, M. (1927/2004). El ser y el tiempo. México, D.F.: Fondo de Cultura Económica.

Hernández-Zamora, G. (2009). Escritura académica y formación de maestros. ¿Por qué no acaban la tesis? Tiempo de Educar, 10(19), 11-40. https://r.issu.edu. $\mathrm{do} / \mathrm{l} ? \mathrm{l}=10682 \mathrm{hfE}$

Lacon de De Lucia, N., \& Ortega de Hocevar, S. (2008). Cognición, metacognición y escritura. Revista signos, 41(67), 231-255.

https://dx.doi.org/10.4067/S0718-09342008000200009

Maingueneau, D. (2002). Problèmes d'ethos. Pratiques, 113(1), 55-67.

Mendes, I. A., \& Silva, C. A. F. da. (2017). Problematização de práticas socioculturais na formação de professores de Matemática. Revista Exitus, 7(2), 100-126.

https://doi.org/10.24065/2237-9460.2017v7n2ID303

Moyano, E. I. (2018). La enseńanza de la lectura y la escritura académicas mediante programas a lo largo del curriculum universitario: opción teórica, didáctica y de gestión. DELTA: Documentação de Estudos em Lingüistica Teórica e Aplicada, 34(1), 235-267. https://dx.doi.org/10.1590/0102-445074896274115057 Nothstein, S. B., \& Valente, E. A. (2016). La producción de escritos en postgrados internacionales. La incidencia de las tradiciones académicas en la apropiación y producción de saberes. Revista Signos. Estudios de Lingüistica, 49(S1) 127-148.

https://dx.doi.org/10.4067/S0718-09342016000400007

Reichenbach, H. (1961). Experience and prediction. An analysis of the foundations and the structure of knowledge. Chicago, Illinois: Phoenix Books the University of Chicago Press.

Roa-Rodríguez, P. (2014). Los textos académicos. Un reto para docentes y estudiantes. Sophia, 10(2),7076. https://r.issu.edu.do/l?l=10686vI5

Rodríguez, M. (2013). Dificultades de aprendizaje relacionadas con la escritura. Pautas para padres y madres. Madrid: CEAPA.

Rodríguez-Arocho, W. C. (2018). Tecnologías de la información y la comunicación: nuevas configuraciones mentales y sus implicaciones para la educación. Revista de Psicología, 27(1), 1-12.

http://dx.doi.org/10.5354/0719-0581.2018.50751

Rodríguez-Arocho, W. C. (2020). Evaluación psicoeducativa y mediación pedagógica: experiencias de integración en Puerto Rico. Actualidades Investigativas en Educación, 20(3), 92-120.

https://r.issu.edu.do/l?l=10687lgx

Rodríguez-Hernández, B. A., \& Leal Vera, R. A. (2017). La escritura académica en los posgrados profesionalizantes para maestros de educación básica. CPU-e. Revista de Investigación Educativa, (24), 224239. https://r.issu.edu.do/l?l=10688sDj 


\section{Revista Caribeña de Investigación Educativa | 2021, 5(2), 68-80}

Rogoff, B. (1993). Aprendices del pensamiento. El desarrollo cognitivo en el contexto social. Madrid: Paidós.

Sosa, R. (2010). Creatividad en la plástica: historia de la construcción social del sujeto creativo. Nomadias, (12). http://dx.doi. org/10.5354/0719-0905.2010.15254

Soto-Figueredo, C. H. (2020). Factores asociados a la elaboración de tesis en la Universidad Nacional de Asunción, Paraguay. Actualidades Investigativas en Educación, 20(2), 500-527.

https://dx.doi.org/10.15517/aie.v20i2.41676

Triana-Murillo, L. M. (2015). Diagnóstico de las competencias lectoescritas en estudiantes de posgrado. Revista Academia y Virtualidad, 8(1), 76-87. https://r.issu.edu.do/l?l=106899Xa
Valarino, E. (1991). El síndrome TMT. Todo, menos Tesis. Posgrado, 1(1), 63-78.

Villegas, M. \& Hernández, M. (2017). La indagación dialógica (ID): una estrategia para la co-formación de docentes en servicio. Revista de la Escuela de Ciencias de la Educación de la Universidad Nacional del Rosario 1(12), 97-113.

https://r.issu.edu.do/l?1=10831eUI

Vygotski, L. S. (1993). Pensamiento y lenguaje. En Lev S. Vygotski: Obras escogidas, tomo II, (pp. 9-348). Madrid: Aprendizaje Visor.

Vygotski, Lev. (1996). El desarrollo de los procesos psicológicos superiores. Barcelona: Crítica.

Wells, G. (2004). Dialogic Inquiry. Towards a Sociocultural Practice and Theory of Education. Cambridge: Cambridge University Press.

\section{CÓMO CITAR:}

Villegas, M. M., \& González, F. (2021). Acompañamiento mediacional para desarrollar la competencia escritual de estudiantes de maestría. RECIE. Revista Caribeña de Investigación Educativa, 5(2), 68-80. https://doi.org/10.32541/recie.2021.v5i2.pp68-80 